\title{
Chiral vortical conductivity across a topological phase transition from holography
}

\author{
Xuanting $\mathrm{Ji}^{1, \$}$ Yan Liu $\odot,{ }^{2,3, \dagger}$ and Xin-Meng $\mathrm{Wu}^{2,3, *}$ \\ ${ }^{1}$ School of Physical Sciences, University of Chinese Academy of Sciences, Beijing 100049, China \\ ${ }^{2}$ Center for Gravitational Physics, Department of Space Science, Beihang University, \\ Beijing 100191, China \\ ${ }^{3}$ Key Laboratory of Space Environment Monitoring and Information Processing, \\ Ministry of Industry and Information Technology, Beijing 100191, China
}

(Received 16 September 2019; published 11 December 2019)

\begin{abstract}
We study the chiral vortical conductivity in a holographic Weyl semimetal model which describes a topological phase transition from the strongly coupled topologically nontrivial phase to a trivial phase. We focus on the temperature dependence of the chiral vortical conductivity where the mixed gaugegravitational anomaly plays a crucial role. After a proper renormalization of the chiral vortical conductivity by the anomalous Hall conductivity and temperature squared, we find that at low temperatures, in both the Weyl semimetal phase and the quantum critical region, this renormalized ratio remains a universal constant. Furthermore, we provide numerical evidence that in the quantum critical region, this ratio depends only on the emergent Lifshitz scaling exponent at the quantum critical point.
\end{abstract}

DOI: $10.1103 /$ PhysRevD.100.126013

\section{INTRODUCTION}

Quantum anomaly induced macroscopic transport physics has attracted much attention recently. On the one hand, the anomalous transports can be studied from many different theoretical approaches, including quantum field theory, chiral kinetic theory, the hydrodynamical method, and holographic methods, etc. Most interestingly, universal behaviors for these anomalous transports are found to be completely determined by the anomaly coefficients, including chiral magnetic effect and chiral vortical effect $[1,2]$. On the other hand, the anomalous transports play a crucial role in the dynamics of many different real physical systems involving chiral fermions, ranging from the quark gluon plasma created at the Relativistic Heavy Ion Collider (RHIC) and Large Hadron Collider (LHC) [3], to neutron stars [4,5], to the more recently discovered Weyl semimetals $[2,6]$.

Mixed gauge-gravitational anomaly is one of the novel quantum anomalies, which is the gravitational contribution to the axial anomaly. At finite temperature, the mixed gaugegravitational anomaly contributes to the chiral vortical

\footnotetext{
*Corresponding author.

wu_xm@buaa.edu.cn

Fyanliu@buaa.edu.cn

\#ixuanting14@mail.ucas.ac.cn
}

Published by the American Physical Society under the terms of the Creative Commons Attribution 4.0 International license. Further distribution of this work must maintain attribution to the author(s) and the published article's title, journal citation, and DOI. Funded by SCOAP. conductivity [7]. More precisely, for a chiral many-body system at finite temperature, the axial current is generated along the direction of rotation in the form of $\mathbf{J}_{5}=\sigma_{V} \boldsymbol{\omega}$ with $\sigma_{V}=c_{g} T^{2}$ and $c_{g}=32 \pi^{2} \zeta$ where $\zeta$ is the coefficient of the gravitational contribution to the axial anomaly $\partial_{\mu} J_{5}^{\mu}=$ $\zeta \epsilon^{\mu \nu \rho \sigma} R^{\alpha}{ }_{\beta \mu \nu} R^{\beta}{ }_{\alpha \rho \sigma}[7,8]$. An incomplete list on the developments of the studies on chiral vortical conductivity is [9-21]. It was reported recently in [22] that the effect of positive longitudinal magneto-thermoelectric conductivity linked to mixed gauge-gravitational anomaly has been observed in the Weyl semimetal NbP.

Most of the previous studies on chiral vortical conductivity focused on systems with time-reversal symmetry. However, in Weyl semimetals where low energy excitations behave as chiral fermions, the time-reversal symmetry could be explicitly broken by the separation of the Weyl nodes, and there might be novel effects on chiral vortical conductivity. Furthermore, Weyl semimetals can go through a topological phase transition to a topological trivial phase. In the critical regime and the trivial phase, since there is no obvious notion of chiral symmetry from the weakly coupled field theory, the computation of chiral vortical conductivity, as well as other anomalous transports, might be a challenging work. Nevertheless, it is still expected that the chiral vortical conductivity shows some interesting behaviors across the phase transition.

The calculation of anomalous transports might be easier for strongly coupled Weyl semimetal. A candidate for strongly coupled Weyl semimetals in the laboratory has been recently reported in [23]. The goal of this work is to investigate the chiral vortical effect induced from the mixed 
gauge-gravitational anomaly in strongly interacting Weyl semimetals. The strongly coupled Weyl semimetal can be studied within the framework of holography [24,25], in which the Ward identities of the dual system are the same as those from weakly coupled field theory, although there is no notion of band structure. It was shown in $[24,25]$ that in the holographic Weyl semimetal model, there is a quantum phase transition between the topologically nontrivial phase and a trivial phase by tuning the ratio between the mass parameter and the time-reversal symmetry breaking parameter. Other aspects of the holographic Weyl semimetal have been explored in [26-33]. A recent review on the holographic Weyl semimetals can be found in [34].

In the following we use Kubo formulas to calculate the chiral vortical conductivity in different phases of holographic Weyl semimetals. Because of the fact that the axial gauge field is screened in the background of a scalar field, the anomalous Hall conductivity and chiral vortical conductivity should have the same normalization factor. ${ }^{1}$ For this reason we use the anomalous Hall conductivity to normalize the chiral vortical conductivity and study the universality of this renormalized quantity in different phases. We also comment on the behavior of odd (Hall) viscosity in this paper.

This paper is organized as follows. We first review the holographic Weyl semimetal model to fix the conventions in Sec. II. In Sec. III, we calculate the chiral vortical conductivity and study its universality upon normalization by the anomalous Hall conductivity. We conclude with a discussion in Sec. IV. The details of the calculation are given in Appendixes $\mathrm{A}$ and $\mathrm{B}$.

\section{SETUP}

We start from the holographic Weyl semimetal model $[24,25]^{2}$ which is described by the following action:

$$
\begin{aligned}
S= & \int d^{5} x \sqrt{-g}\left[\frac{1}{2 \kappa^{2}}\left(R+\frac{12}{L^{2}}\right)-\frac{1}{4 e^{2}} \mathcal{F}^{2}-\frac{1}{4 e^{2}} F^{2}\right. \\
& +\epsilon^{a b c d e} A_{a}\left(\frac{\alpha}{3}\left(F_{b c} F_{d e}+3 \mathcal{F}_{b c} \mathcal{F}_{d e}\right)+\zeta R_{n b c}^{m} R_{m d e}^{n}\right) \\
& \left.-\left(D_{a} \Phi\right)^{*}\left(D^{a} \Phi\right)-V(\Phi)\right]
\end{aligned}
$$

where $2 \kappa^{2}$ is the five-dimensional gravitational coupling constant and $L$ is the AdS radius. We set $2 \kappa^{2}=L=1$ from now on. Two $U(1)$ gauge fields $V_{a}$ and $A_{a}$ are dual to the vector current and the axial current, respectively, and their field strengths are

$$
\mathcal{F}_{a b}=\partial_{a} V_{b}-\partial_{b} V_{a}, \quad F_{a b}=\partial_{a} A_{b}-\partial_{b} A_{a} .
$$

\footnotetext{
${ }^{1}$ More details on this point will be discussed in Sec. III A.

${ }^{2}$ Note that $\epsilon_{a b c d e}=\sqrt{-g} \varepsilon_{a b c d e}$ with $\varepsilon_{0123 r}=1$.
}

The Chern-Simons terms are introduced to give the correct anomalous structure for the dual field theory, including $U(1)_{A}^{3}$ and $U(1)_{A} U(1)_{V}^{2}$ anomaly and mixed gaugegravitational anomaly. The scalar field $\Phi$ is axially charged and has the potential

$D_{a} \Phi=\left(\partial_{a}-i q A_{a}\right) \Phi, \quad V(\Phi)=m^{2}|\Phi|^{2}+\frac{\lambda}{2}|\Phi|^{4}$.

We choose $m^{2}=-3, \lambda=1 / 2, q=4 / 5$ without loss of generality in this paper. $^{3}$

The ansatz for the background at finite temperature is

$$
\begin{aligned}
d s^{2} & =-u d t^{2}+\frac{d r^{2}}{u}+f\left(d x^{2}+d y^{2}\right)+h d z^{2}, \\
A & =A_{z} d z, \quad \Phi=\phi(r),
\end{aligned}
$$

where $u, f, h, A_{z}, \phi$ are functions of the radial coordinate $r$. The equations of motion for these fields can be found in [24]. From the conserved quantity $\left(\sqrt{h}\left(u^{\prime} f-u f^{\prime}\right)\right)^{\prime}=0$, one concludes $f=u$ at zero temperature. This is also consistent with the intuition that there is an $S O(1,2)$ symmetry along the $t, x, y$ directions for the zero-temperature dual field theory. Close to the UV boundary, we have $\lim _{r \rightarrow \infty} A_{z}=b$ and $\lim _{r \rightarrow \infty} r \phi=M$, which are the timereversal symmetry breaking parameter and the axial symmetry breaking parameter separately. The details of the expansion for the background fields near the boundary can be found in Appendix A.

At zero temperature there exist three different kinds of near horizon solutions, which flow to three different regimes of $M / b$ at the UV boundary [24]. In the following, we briefly summarize these three kinds of IR solutions near the horizon and highlight the emergent symmetry of the Lifshitz-type critical solution.

Weyl semimetal phase: At the leading order the first type of IR solution is an $\mathrm{AdS}_{5}$ with nonvanishing axial gauge field $A_{z}(r=0)$. Including the subleading correction, it takes the form

$$
\begin{aligned}
& u=r^{2}, \quad h=r^{2}, \quad A_{z}=a_{1}+\frac{\pi a_{1}^{2} \phi_{1}^{2}}{16 r} e^{-\frac{2 a_{1} q}{r},} \\
& \phi=\sqrt{\pi} \phi_{1}\left(\frac{a_{1} q}{2 r}\right)^{3 / 2} e^{-\frac{a_{1} q}{r}},
\end{aligned}
$$

where $a_{1}, \phi_{1}$ are the parameters for the IR geometry that are related to the parameters $M$ and $b$ for the UV geometry via RG flow. This type of IR geometry can flow to UV with $M / b<0.906$ for $\lambda=1 / 2, q=4 / 5$.

\footnotetext{
${ }^{3}$ In Sec. III B, we switch the parameters $\lambda$ and $q$ while keeping the scaling exponent fixed to study the universality of the anomalous transports after a proper renormalization in the critical region.
} 


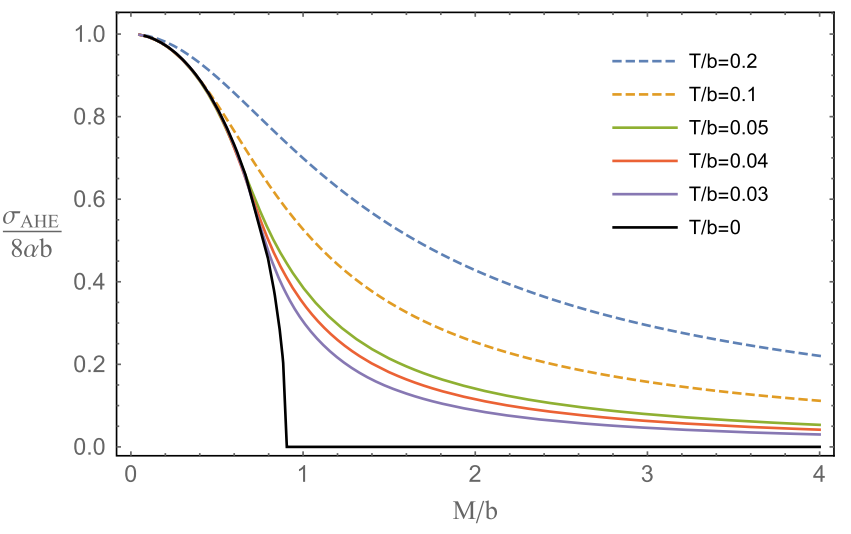

FIG. 1. Anomalous Hall conductivity as a function of $M / b$ at zero and finite temperatures in the holographic Weyl semimetal for $m^{2}=-3, \lambda=1 / 2, q=4 / 5$. High $(T / b=0.2,0.1)$, low $(T / b=0.05,0.04,0.03)$, and zero temperatures $(T / b=0)$ are shown in dashed, solid, and thick lines, respectively.

Quantum critical point: At the leading order, the second type of IR solution is

$$
\begin{aligned}
d s^{2} & =u_{0} r^{2}\left(-d t^{2}+d x^{2}+d y^{2}\right)+\frac{d r^{2}}{u_{0} r^{2}}+h_{0} r^{2 \beta} d z^{2}, \\
A_{z} & =r^{\beta}, \quad \phi=\phi_{0} .
\end{aligned}
$$

With irrelevant perturbation, the IR geometry can flow to $\mathrm{UV}$, and it turns out in UV $(M / b)_{c} \simeq 0.906$, which corresponds to the critical point in the topological quantum phase transition [24]. This critical solution has anisotropic Lifshitz-type symmetry under the scaling transformation $\left(t, x, y, r^{-1}\right) \rightarrow s\left(t, x, y, r^{-1}\right)$ and $z \rightarrow s^{\beta} z$. There are four numerical values $\left\{u_{0}, h_{0}, \beta, \phi_{0}\right\}$ that are determined by the value of $\lambda, m$, and $q$. In particular, $\beta$ depends on two parameters $q, \lambda$ as

$$
\beta=-\frac{2 q^{2}}{-3-2 q^{2}+\lambda \phi_{0}^{2}},
$$

where $\phi_{0}$ depends on $q, \lambda$ in a complicated way. Therefore, we can continuously tune $q$ and $\lambda$ to obtain different critical solutions which have the same emergent Lifshitz scaling exponent $\beta$. This makes it possible to study the relations between the anomalous transports and the emergent Lifshitz scaling exponent. Furthermore, the null-energy condition constrains that $\beta \leq 1$, and regularity of solutions demands that $\beta>0$, i.e., $\beta \in(0,1]$ [29].

Topological trivial phase: The third type of IR solution at the leading order is an $\mathrm{AdS}_{5}$ with nonvanishing $\phi$. It takes the form

$$
\begin{aligned}
u & =\left(1+\frac{3}{8 \lambda}\right) r^{2}, \quad h=r^{2}, \quad A_{z}=a_{1} r^{\beta_{1}}, \\
\phi & =\sqrt{\frac{3}{\lambda}}+\phi_{1} r^{\beta_{2}}
\end{aligned}
$$

where $\left(\beta_{1}, \beta_{2}\right)=\left(\sqrt{1+\frac{48 q^{2}}{3+8 \lambda}}-1,2 \sqrt{\frac{3+20 \lambda}{3+8 \lambda}}-2\right)$. This type of IR geometry can flow to the asymptotic AdS boundary with $M / b>0.906$.

The anomalous Hall conductivity in these three different regimes behaves as shown in the plot of Fig. 1. For $T / b=0$, the discontinuity of $\sigma_{\mathrm{AHE}} / b$ indicates that there is a quantum phase transition at the quantum critical point (QCP) $M_{c} / b \simeq 0.906$. At finite temperatures $T / b>0$, the $\mathrm{QCP}$ is enlarged to a quantum critical region (QCR). As a result, the sharp line becomes smooth crossovers. In the Weyl semimetal phase, $\sigma_{\mathrm{AHE}} / b$ is independent of $T / b$ at low temperatures.

\section{CHIRAL VORTICAL CONDUCTIVITY AND UNIVERSALITY}

In this section, we study the chiral vortical conductivity at finite temperature in the holographic model (2.1). We first focus on the crossover of chiral vortical conductivity across the topological phase transition at different temperatures. From the field theory point of view, this is to investigate how the fermion mass influences the chiral vortical effect in a time-reversal symmetry breaking system. Note that we concentrate on the contribution induced from the mixed gauge-gravitational anomaly. In Sec. III B, we study the physics in the critical region, including the behaviors of the chiral vortical conductivity and odd (Hall) viscosities. Since different critical ground states could have the same emergent scaling exponent $\beta$ of the Lifshitz symmetry, as explained at footnote 3 , we verify the possible existence of universal physics in the critical region.

\section{A. Chiral vortical conductivity}

The chiral vortical conductivity for the dual field theory of the holographic model can be computed from the Kubo formula

$$
\sigma_{V}=\left.\lim _{k_{l} \rightarrow 0} \frac{i}{2 k_{l}} \sum_{m, n} \epsilon_{l m n}\left\langle J_{5}^{n} T^{t m}\right\rangle\right|_{\omega=0},
$$

where $m, n, l \in\{x, y, z\}, J_{5}$ is the axial current, and $T^{\mu \nu}$ is the energy momentum tensor. The holographic Weyl semimetal is an anisotropic system, and we have two independent chiral vortical conductivities. For simplicity, we focus on the component with fluctuation along the $z$ direction ${ }^{4}$; i.e., we choose the $k_{z} \rightarrow 0$ limit in (3.1).

We switch on the following perturbations:

$$
\delta g_{t i}=h_{t i} e^{i k_{z} z}, \quad \delta g_{i z}=h_{i z} e^{i k_{z} z}, \quad \delta A_{i}=a_{i} e^{i k_{z} z}
$$

\footnotetext{
${ }^{4}$ To consider the other component of the chiral vortical conductivity, we need to turn on all the fluctuations around the background to do the calculation. This is extremely complicated and beyond the scope of this work.
} 
for $i \in\{x, y\}$. With six perturbation fields under consideration, one obtains rather intractable equations of motion for $\delta A_{i}$ as the corresponding response to the source $\delta g_{t j}$. The details of equations can be found in Appendix B. For all the fields, we expand as, e.g., $a_{i}=a_{i}^{(0)}+k_{z} a_{i}^{(1)}+\cdots$, and we solve the equations order by order in $k_{z}$. At zeroth order, the solutions are $h_{t i}^{(0)}=u$ and $a_{i}^{(0)}=0$. At first order, $h_{t i}^{(1)}=0$, and the equation of motion for $a_{i}^{(1)}$ is

$$
\begin{gathered}
a_{i}^{(1) \prime \prime}+\left(\frac{u^{\prime}}{u}+\frac{h^{\prime}}{2 h}\right) a_{i}^{(1) \prime}-\left(\frac{2 q^{2} \phi^{2}}{u}-\frac{A_{z}^{\prime 2}}{h}\right) a_{i}^{(1)} \\
-\frac{2 i \zeta}{\sqrt{h}} \epsilon_{i j}\left(\frac{u^{\prime}}{u}-\frac{f^{\prime}}{f}\right) L_{1}=0,
\end{gathered}
$$

with

$$
\begin{aligned}
L_{1}= & {\left[-\left(\frac{2 h^{\prime}}{h}+\frac{f^{\prime}}{f}\right) h_{t j}^{(0) \prime}+\left(\left(\frac{f^{\prime \prime}}{f}-\frac{h^{\prime \prime}}{h}\right)\right.\right.} \\
& \left.\left.+\frac{3 h^{\prime}}{2 h}\left(\frac{f^{\prime}}{f}+\frac{h^{\prime}}{h}\right)+\frac{A_{z}^{\prime 2}}{h}\right) h_{t j}^{(0)}\right],
\end{aligned}
$$

where $\epsilon_{x y}=-\epsilon_{y x}=1$. Note that this equation of motion decouples from $h_{i z}$. We make some observations here before discussing the numerical results:

(i) When $T=0, u=f$, the chiral vortical conductivity vanishes. In other words, at zero temperature, the rotation cannot induce any axial current.

(ii) At finite temperature, $\sigma_{V} \propto \zeta$. This indicates that the chiral vortical effect in this system is completely induced from the mixed gauge-gravitational anomaly.

(iii) When $\phi=0$, we have $A_{z}=b, f=h=r^{2}$, and $u=r^{2}\left(1-\frac{r_{0}^{4}}{r^{4}}\right)$. This is exactly the Schwarzschild background with a constant $A_{z}$. The equation of motion (3.3) can be solved analytically. From Eq. (3.3), one obtains

$$
a_{i}^{(1)}=\int_{r_{0}}^{r} d \tilde{r}\left[\frac{32 i \zeta r_{0}^{8}}{u \sqrt{h} \tilde{r}^{6}}-\frac{32 i \zeta r_{0}^{2}}{u \sqrt{h}}\right] .
$$

Therefore, $a_{i}^{(1)}=\frac{16 i \zeta r_{0}^{2}}{r^{2}}$ near the boundary with $r_{0}=\pi T$, and one obtains $\sigma_{V}=32 \pi^{2} \zeta T^{2}$. This is exactly the same as the results in $[7,8]$ in the zero density limit.

(iv) For the generic case with $\phi \neq 0$, there is not an analytical bulk solution. The numerical tool is necessary to obtain the chiral vortical conductivity.

The numerical approach to solving (3.3) is summarized as follows. First, we numerically obtain the background solution [27]. Starting from the near horizon geometry, one can integrate to the boundary to obtain the whole geometry in the bulk. Because of the scaling symmetries, there are two free shooting parameters, which correspond to $M / b$

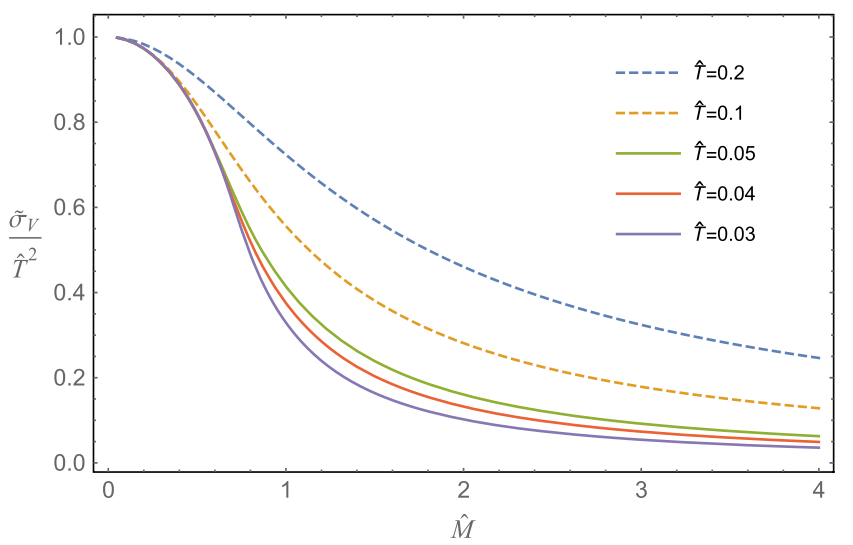

FIG. 2. The dependence of the chiral vortical conductivity $\tilde{\sigma}_{V}$ over $\hat{T}^{2}$ on $\hat{M}$ at different temperatures. Here, $\tilde{\sigma}_{V} / \hat{T}^{2}$ exhibit similar behavior with respect to $\tilde{\sigma}_{\mathrm{AHE}}$ as a function of dimensionless $\hat{M}$.

and $T / b$ in the dual field theory. Then with the background solution obtained for given $M / b$ and $T / b$, Eq. (3.3) can be solved with the appropriate regular boundary condition at the horizon and the sourceless boundary condition at the conformal boundary. The details to obtain the Green's function can be found in Appendix B.

For convenience, we define the dimensionless mass, the dimensionless temperature, and the dimensionless (normalized) conductivities as

$$
\begin{aligned}
\hat{M} & \equiv \frac{M}{b}, \quad \hat{T} \equiv \frac{T}{b}, \quad \tilde{\sigma}_{\mathrm{AHE}} \equiv \frac{\sigma_{\mathrm{AHE}}}{8 \alpha b}, \\
\tilde{\sigma}_{V} & \equiv \frac{\sigma_{V}}{32 \pi^{2} \zeta b^{2}} .
\end{aligned}
$$

In Fig. 2, we numerically demonstrate the reduced chiral vortical conductivity over temperature squared $\tilde{\sigma}_{V} / \hat{T}^{2}$ as a function of $\hat{M}$ at various temperatures. When $\hat{M} \rightarrow 0$, one obtains $\sigma_{V}=32 \pi^{2} \zeta T^{2}$ which is the case where we do not explicitly break the chiral symmetry $[7,8]$. When $\hat{M}$ increases, the chiral vortical conductivity decreases. Within the Weyl semimetal phase, as the temperature is sufficiently low $(\hat{T} \leq 0.05), \tilde{\sigma}_{V} / \hat{T}^{2}$ shows a perfect coincidence, similar to the anomalous Hall conductivity in Fig 1. This means that $\tilde{\sigma}_{V} \propto \hat{T}^{2}$ in the Weyl semimetal phase. In the quantum critical region and the topologically trivial phase, the chiral vortical conductivity decreases monotonically and approaches zero as a result of the degrees of freedom of chiral fermions being gradually gapped out. It is interesting to note that Fig. 2 shows the mass dependence of $\tilde{\sigma}_{V} / \hat{T}^{2}$ of the strongly coupled massive fermion system, which behaves qualitatively the same as the investigation of the mass effect of the chiral vortical effect in the weakly coupled massive fermion system [35,36]. We should emphasize that in our studies, time-reversal symmetry is broken while this symmetry is preserved in $[35,36]$. 

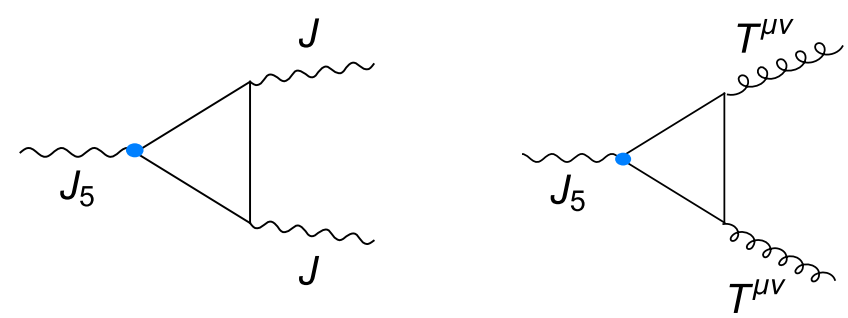

FIG. 3. Feynman diagrams of the leading-order corrections to the two-point correlators $\langle J J\rangle$ and $\left\langle J_{5} T\right\rangle$. These are the most significant contributions when computing the anomalous Hall (left plot) and the chiral vortical (right plot) conductivities. The blue dot in each diagram represents the renormalized factor that is proportional to $\sqrt{Z_{A}}$. Both the diagrams scale as $\sqrt{Z_{A}}$.

Moreover, from Figs. 1 and 2, one may notice that $\tilde{\sigma}_{V} / \hat{T}^{2}$ shows very similar behavior compared to $\tilde{\sigma}_{\mathrm{AHE}}{ }^{5}$ Although both of these two conductivities approach zero at large $\hat{M} \gg \hat{M}_{c}$, the ratio of these two conductivities shows nontrivial physics. The reason to investigate the normalized ratio of $\tilde{\sigma}_{V} / \hat{T}^{2}$ to $\tilde{\sigma}_{\mathrm{AHE}}$ is motivated by the suggestion made in [29] that when we compute the two-point correlation functions, the proper renormalization effect of the axial current should be taken into account. In other words, when we consider the physics at a low energy scale near the Fermi surface, the dc conductivities will be related to the IR coupling of the external fields, which is, in general, different from the UV strength. Following [29] we introduce a renormalized factor $\sqrt{Z_{A}}$ satisfying

$$
\sqrt{Z_{A}} b=b^{\mathrm{IR}}
$$

where

$$
b=\lim _{r \rightarrow \infty} A_{z}, \quad b^{\mathrm{IR}}=A_{z}\left(r_{0}\right) .
$$

This coupling renormalization has impacts on both the anomalous Hall conductivity $\tilde{\sigma}_{\mathrm{AHE}}$ and the chiral vortical conductivity $\tilde{\sigma}_{V}$, as shown in the leading-order contribution of Feynman diagrams for the anomalous contributions to the two-point correlators $\langle J J\rangle$ and $\left\langle J_{5} T\right\rangle$ in Fig. 3.

Therefore, to uncover the possible universal physics at low energy, we renormalize the chiral vortical conductivity through $\tilde{\sigma}_{\mathrm{AHE}}$. The behavior of $\tilde{\sigma}_{V} /\left(\tilde{\sigma}_{\mathrm{AHE}} \hat{T}^{2}\right)$ as a function of $\hat{M}$ is shown in Fig. 4, from which several universalities regarding to chiral vortical effect could be revealed. First, in the Weyl semimetal phase at low temperatures, the ratio is almost a constant due to the cancellation of infrared screening in both of these two transports. Although with different choices of $\hat{M}$ the underlying bulk geometries have

\footnotetext{
${ }^{5}$ Note that there is one obvious difference: that at zero temperature $\tilde{\sigma}_{V}$ vanishes in all three phases, while $\tilde{\sigma}_{\text {AHE }}$ does not vanish in the Weyl semimetal phase.
}

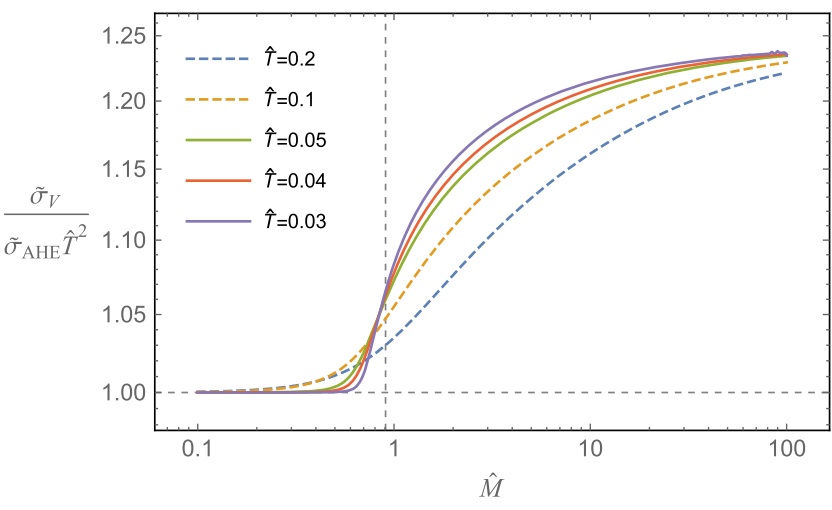

FIG. 4. The normalized chiral vortical conductivity $\tilde{\sigma}_{V}$ over normalized anomalous Hall conductivity $\tilde{\sigma}_{\mathrm{AHE}}$ and $\hat{T}^{2}$ as a function of dimensionless $\hat{M}$ at different temperatures. The perpendicular grid line at $\hat{M}_{c}=0.906$ indicates the QCP. High temperatures $(\hat{T}=0.2,0.1)$ and low temperatures $(\hat{T}=0.05$, $0.04,0.03)$ are shown as dashed and solid lines, respectively. At low temperature the ratio departs from a constant value in the Weyl semimetal phase, increases in the vicinity of the QCP, and reaches a temperature-independent value at large $\hat{M}$.

different profiles, this ratio is in coincidence with the case $\hat{M}=0$. More interestingly, this property is the same in the topological nontrivial phase for all possible choices of $(q, \lambda)$ that allow a quantum phase transition in this holographic model. Second, in the quantum critical region, $\tilde{\sigma}_{V} / \tilde{\sigma}_{\mathrm{AHE}} \propto \hat{T}^{2}$. More universal behaviors for the mixed anomaly induced transports in this phase will be studied in detail in Sec. III B. Third, at large enough $\hat{M} \gg \hat{M}_{c}$, the ratio approaches another value, which is independent of $\hat{T}$ and is greater than 1 , while this value depends on the choice of $(q, \lambda)$.

\section{B. Universality of mixed anomaly induced transports}

In this subsection, we focus on the universal physics of the mixed gauge-gravitational anomaly induced transports in the quantum critical region.

As shown in Fig. 4, in the vicinity of the QCP located at $\hat{M}_{c} \simeq 0.906, \tilde{\sigma}_{V} /\left(\tilde{\sigma}_{\mathrm{AHE}} \hat{T}^{2}\right)$ tends to exhibit overlaps for a variety of low temperatures. One can clearly find the temperature dependence of the renormalized chiral vortical conductivity from Fig. 6. As is adequately discussed in [27], at low temperature, the scaling behavior of $\tilde{\sigma}_{\mathrm{AHE}}$ in the quantum critical region is determined from the anisotropic scaling exponent $\beta$, i.e., $\tilde{\sigma}_{\mathrm{AHE}} \propto \hat{T}^{\beta}$. However, for $\tilde{\sigma}_{V}$, we cannot analytically express it in terms of the horizon data due to the nonexistence of a radially conserved quantity. Nevertheless, a careful analysis of numerical data shows that there exists an exact power law for the normalized chiral vortical conductivity when $\hat{T} \rightarrow 0$, i.e., $\tilde{\sigma}_{V} \propto \hat{T}^{\gamma_{2}}$. In Fig. 5, we show the temperature scaling exponents $\gamma_{1}, \gamma_{2}, \gamma_{3}$ 


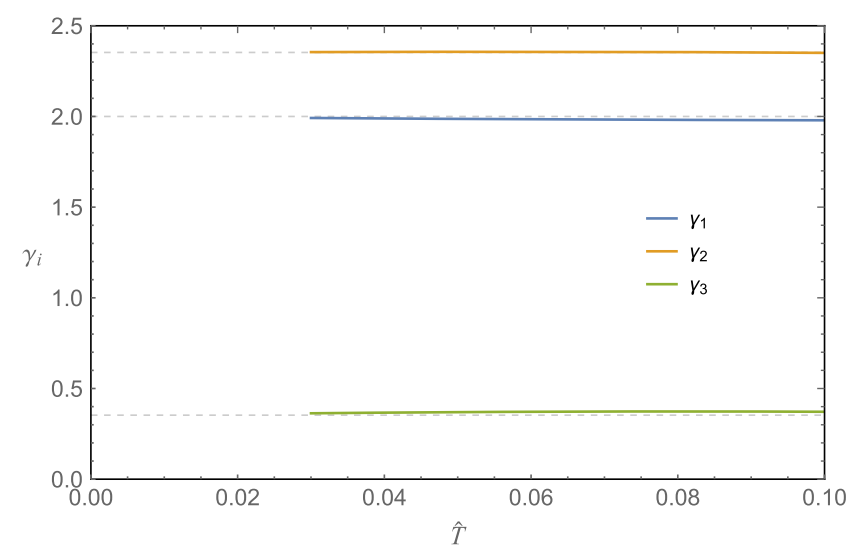

FIG. 5. The temperature scaling exponents $\gamma_{i}$ with $\left\{\gamma_{1}, \gamma_{2}, \gamma_{3}\right\}$ for $\left\{\tilde{\sigma}_{V} / \tilde{\sigma}_{\mathrm{AHE}}, \tilde{\sigma}_{V}, \tilde{\sigma}_{\mathrm{AHE}}\right\}$, respectively, at the critical value $\hat{M}_{c}=$ 0.906 in the low-temperature regime. The three dashed lines from top to bottom denote $\gamma=2+\beta, 2$ and $\beta$ with $\beta=0.353$.

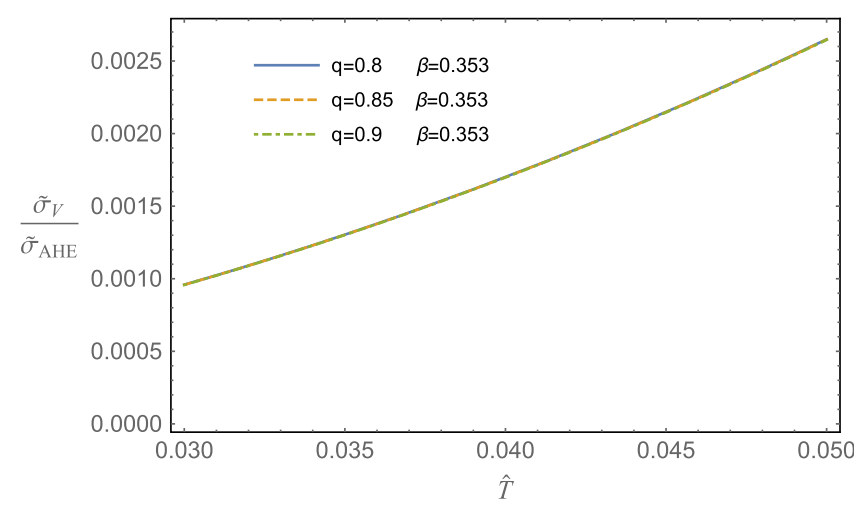

FIG. 6. Universality of $\tilde{\sigma}_{V} / \tilde{\sigma}_{\mathrm{AHE}}$ at low temperatures in the quantum critical region with the IR scaling exponent $\beta=0.353$. With $\beta$ fixed, the choices of $(q, \lambda)$ are $(0.8,0.5)$ (blue), $(0.85,0.308)$ (orange dashed), $(0.9,0.027)$ (green dot-dashed) with $\hat{M}_{c}=0.906$, $0.808,0.730$, respectively. However, $\tilde{\sigma}_{V} / \tilde{\sigma}_{\mathrm{AHE}}$ is independent of the choice of $(q, \lambda)$ and is only a function of $\beta$ and $\hat{T}$.

of $\tilde{\sigma}_{V} / \tilde{\sigma}_{\mathrm{AHE}}, \tilde{\sigma}_{V}$, and $\tilde{\sigma}_{\mathrm{AHE}}$ and find that these three exponents nicely match with $\left(\gamma_{1}, \gamma_{2}, \gamma_{3}\right)=(2,2+\beta, \beta)$.

We also find that the renormalized chiral vortical conductivity exhibits universal behaviors in the quantum critical region as shown in Fig. 6. When we fix the IR scaling exponent $\beta=0.353$ without loss of generality and change the two parameters $q$ and $\lambda$ in this holographic model, the locations of the QCPs $\hat{M}_{c}$ are different. However, $\tilde{\sigma}_{V} / \tilde{\sigma}_{\mathrm{AHE}}$ in the cases with different $(q, \lambda)$, while the same $\beta$ show exactly the same dependence on $\hat{T}$ at low temperatures; however, neither $\tilde{\sigma}_{V}$ nor $\tilde{\sigma}_{\mathrm{AHE}}$ alone behaves in the same way. Therefore, numerical evidence strongly suggests that

$$
\frac{\tilde{\sigma}_{V}}{\tilde{\sigma}_{\mathrm{AHE}}}=c(\beta) \hat{T}^{2}
$$

in the quantum critical region, where $c(\beta)$ is introduced as a coefficient function of the scaling exponent $\beta$.

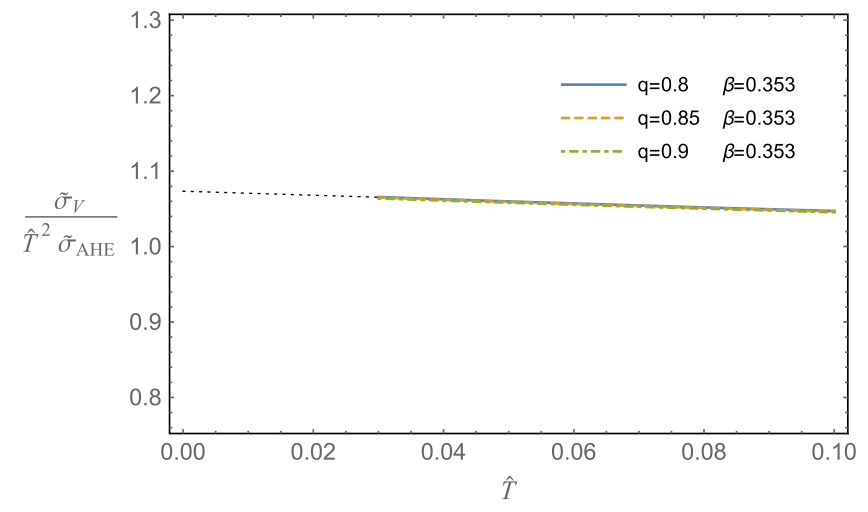

FIG. 7. The coefficient function $c(\beta)$ at low temperatures for $\beta=0.353$. One finds that at low temperatures $c(\beta=0.353)$ is almost a constant. The black dashed line is the interpolation of $c(\beta)$ to $\hat{T}=0$ from which we can get the numerical error between $\left.c\right|_{\hat{T}=0.03}$ and $\left.c\right|_{\hat{T} \rightarrow 0}$.

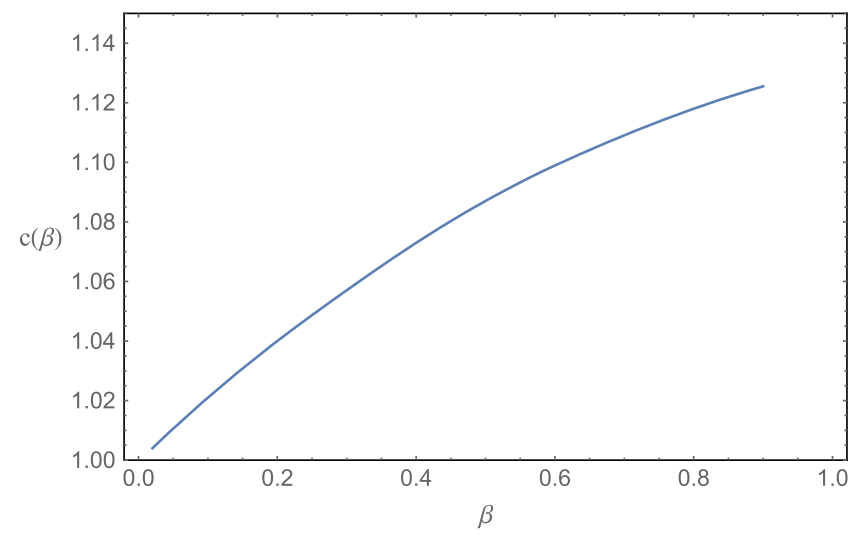

FIG. 8. We show $c(\beta)$ defined in (3.9) as a function of the anisotropic scaling exponent $\beta$ in the quantum critical region.

With fixed $\beta=0.353$, one can study the behavior of $c(\beta)$ at low temperatures as shown in Fig. 7. Because of numerical difficulties, the temperature $\hat{T}$ cannot be arbitrarily small. Figure 7 shows that this coefficient function $c(\beta)$ changes very slowly with a negative slope at about $\Delta c / \Delta \hat{T} \approx-0.27$. From a linear interpolation as shown by the black dashed line in Fig. 7 , one can read $\left.c\right|_{\hat{T} \rightarrow 0}$, from which one gets the numerical difference between $\left.c\right|_{\hat{T}=0.03}$ and $\left.c\right|_{\hat{T} \rightarrow 0}$,

$$
\frac{\left.c\right|_{\hat{T}=0.03}-\left.c\right|_{\hat{T} \rightarrow 0}}{\left.c\right|_{\hat{T}=0.03}} \simeq-0.8 \%
$$

for $\beta=0.353$. We have checked that the numerical error is always small for $\beta \in(0,1)$. This indicates that it is reasonable to obtain this coefficient function $c(\beta)$ at

\footnotetext{
${ }^{6}$ This coefficient function is not well defined at zero temperature where chiral vortical conductivity vanishes. Also, $\left.c\right|_{\hat{T} \rightarrow 0}$ is obtained from fitting the existing data as shown by the black dashed line in Fig. 7.
} 

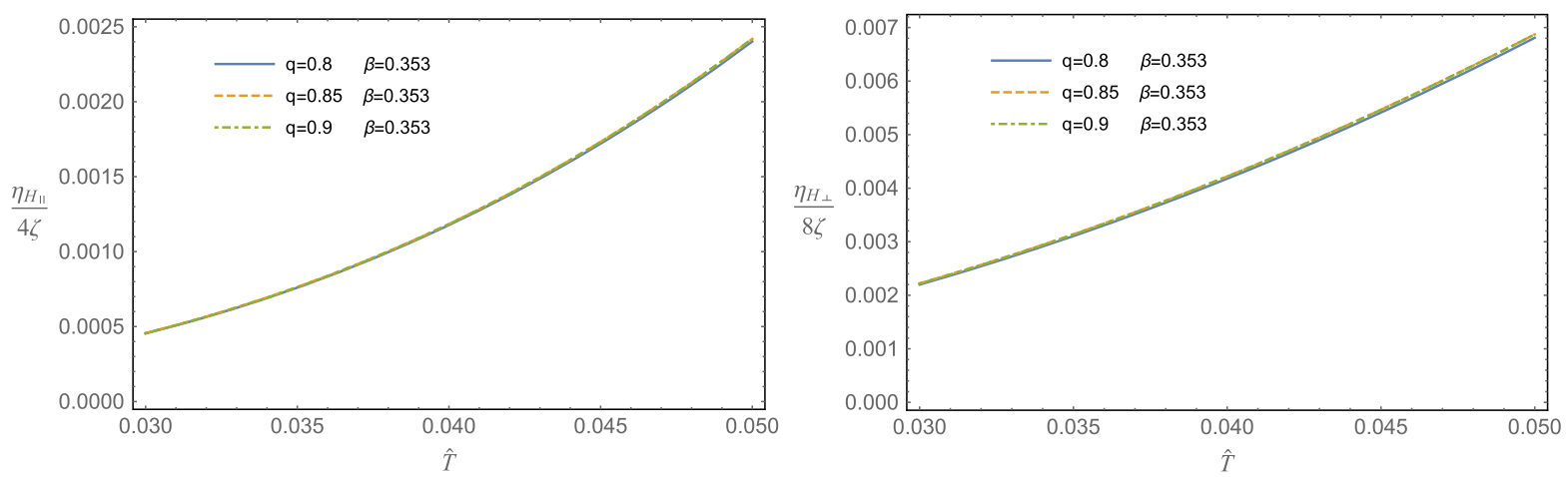

FIG. 9. Universality of odd viscosities $\eta_{H_{\|}}$and $\eta_{H_{\perp}}$ at different QCPs with the same $\beta=0.353$. These two mixed anomaly induced viscosities only depend on the scaling factor $\beta$ in the quantum critical region.

$\hat{T}=0.03$, given that the solutions are inaccessible at too low temperatures.

Because of the extremely small numerical error (3.10), we expect that the value $c(\beta)$ is universal at low temperature, and it does not depend on the details of the dual theory; it only depends on the Lifshitz exponent $\beta$ at low energy. In Fig. 8, we numerically show the $\beta$ dependence of this coefficient function $c(\beta)$ in a series of strongly coupled Lifshitz-like quantum critical systems. $c(\beta)$ monotonically increases with respect to $\beta .^{7}$ It would be very intriguing to compute $c(\beta)$ from kinetic theory and hydrodynamics to compare to this numerical result from the holographic approach.

It is expected that similar universalities exist for other mixed gauge-gravitational anomaly induced transports such as odd (Hall) viscosities $\eta_{H_{\|}}$and $\eta_{H_{\perp}}$ in the quantum critical region, as is shown in Fig. 9. Different from the chiral vortical conductivity, these two odd viscosities can be obtained directly from the near-horizon geometry [27]. In addition to the universal behaviors relying on the anisotropic scaling exponent $\beta$ as $\hat{T} \rightarrow 0$, one can also notice that as the temperature is increased, these behaviors gradually disappear. This can be viewed as evidence that the thermal effect depresses the quantum effect. One can again examine the coefficient functions from these two odd viscosities. However, different from the chiral vortical conductivity, the related numerical errors for odd viscosities are much larger in the regime of the temperature we considered, which makes numerical results not very useful. It is expected that when the temperature is sufficiently low, the numerical error will be under control, and there will be similar universalities

\footnotetext{
${ }^{7}$ The odd (Hall) viscosity has been studied recently in [37] by considering the field theory for critical Lifshitz fermions at finite temperature, which is different from our study where the Lifshitz symmetry is an exact symmetry only at zero temperature in the IR fixed point. In our study, there will always be a small deviation from Lifshitz symmetry due to some irrelevant deformations at low and finite temperature.
}

in the coefficient functions as in the case of chiral vortical conductivity. We will not discuss them here.

\section{CONCLUSIONS}

We have studied the chiral vortical conductivity in a holographic Weyl semimetal model [24] in which there exists a quantum phase transition between the strongly coupled topologically nontrivial phase and a trivial phase. Motivated by [29], we renormalized the chiral vortical conductivity by the anomalous Hall conductivity and temperature squared, and explored the universal physics of this ratio at low temperature. In the Weyl semimetal phase, we found that at low temperature the renormalized chiral vortical conductivity $\tilde{\sigma}_{V} /\left(\tilde{\sigma}_{\mathrm{AHE}} \hat{T}^{2}\right)$ stays constant. In the quantum critical region, the renormalized chiral vortical conductivity is also a constant value, which only depends on the IR scaling exponent $\beta$ at the quantum critical point while independent of the microscopic details of the dual theory; e.g., we can get the same constant by choosing different values of $\lambda, q$ which give rise to different $M_{c} / b$ but with the same IR scaling exponent $\beta$. In the topologically trivial phase, for sufficiently large $M / b$ the renormalized chiral vortical conductivity goes to another constant, which typically depends on the details of the theory.

The universality in the quantum critical region is quite intriguing. The numerical evidence from holography indicates that the scaling exponent $\beta$ is the only parameter for the mixed gauge-gravitational anomaly induced chiral vortical conductivity discussed in Sec. III. This convincing evidence from holography might motivate one to consider the role of the mixed gauge-gravitational anomaly in the Lifshitz-like critical systems for weakly coupled theories and also from other computational approaches. A probable alternative approach is to study the quantum field theory consisting of a massive fermion coupled to an axial $U(1)$ field with a background $U(1)_{A}$ field along spatial directions and calculate the chiral vortical conductivity and the anomalous Hall conductivity at finite temperature to study the universalities of these transports. 


\section{ACKNOWLEDGMENTS}

We would like to thank Rong-Gen Cai, Karl Landsteiner, Francisco Pena-Benitez, and Ya-Wen Sun for useful discussions. This work has been supported by the Thousand Young Talents Program of China. The work of X. J. was also supported by the University of Chinese Academy of Sciences through Grant No. Y95402XXX2. The work of Y. L. and X. M. W. was also supported by a grant from Beihang University and the National Natural Science Foundation of China (NSFC) Grant No. 11875083.

\section{APPENDIX A: ASYMPTOTIC EXPANSIONS FOR THE BACKGROUND FIELDS}

Near conformal boundary, the background fields can be expanded as

$$
\begin{aligned}
u & =r^{2}-\frac{M^{2}}{3}+\frac{M^{4}(2+3 \lambda)}{18} \frac{\ln r}{r^{2}}-\frac{M_{b}}{3 r^{2}}+\ldots \\
f & =r^{2}-\frac{M^{2}}{3}+\frac{M^{4}(2+3 \lambda)}{18} \frac{\ln r}{r^{2}}-\frac{f_{3}}{r^{2}}+\ldots \\
h & =r^{2}-\frac{M^{2}}{3}+\left(\frac{M^{4}(2+3 \lambda)}{18}+\frac{q^{2} b^{2} M^{2}}{2}\right) \frac{\ln r}{r^{2}}+\frac{h_{3}}{r^{2}}+\ldots \\
A_{z} & =b-b M^{2} q^{2} \frac{\ln r}{r^{2}}+\frac{\zeta}{r^{2}}+\ldots \\
\phi & =\frac{M}{r}-\frac{\ln r}{6 r^{3}}\left(2 M^{3}+3 b^{2} M q^{2}+3 M^{3} \lambda\right)+\frac{O}{r^{3}}+\ldots
\end{aligned}
$$

\section{APPENDIX B: CALCULATIONS OF CHIRAL VORTICAL CONDUCTIVITY}

We outline the details of calculating the chiral vortical conductivity. Note that in the field theory the chiral vortical conductivity can be obtained by Kubo formulas (3.1). In holography, we consider the following fluctuations:

$$
\delta g_{j z}=\delta g_{z j}=h_{j z}(r) e^{i k_{z} z}, \quad \delta g_{t j}=\delta g_{j t}=h_{t j}(r) e^{i k_{z} z}, \quad \delta A_{j}=a_{j}(r) e^{i k_{z} z},
$$

with $j \in\{x, y\}$. The equations of motion are

$$
\begin{aligned}
h_{t i}^{\prime \prime}+ & \frac{h^{\prime}}{2 h} h_{t i}^{\prime}+C_{1} h_{t i}-\frac{2 i \zeta k_{z}}{\sqrt{h}} \epsilon_{i j}\left[D_{1}\left(h_{j z}^{\prime}-\frac{f^{\prime}}{f} h_{j z}\right)+E_{1} a_{j}^{\prime}+F_{1} a_{j}\right]=0, \\
h_{i z}^{\prime \prime}+ & \left(\frac{u^{\prime}}{u}-\frac{h^{\prime}}{2 h}\right) h_{i z}^{\prime}+\left(\frac{f^{\prime} h^{\prime}}{2 f h}-\frac{f^{\prime \prime}}{f}-\frac{f^{\prime} u^{\prime}}{f u}\right) h_{i z}+A_{z}^{\prime} a_{i}^{\prime}+\frac{2 q^{2} \phi^{2} A_{z}}{u} a_{i} \\
& -\frac{2 i \zeta k_{z}}{\sqrt{h}} \epsilon_{i j}\left[A_{z}^{\prime}\left(\frac{u^{\prime}}{u}-\frac{f^{\prime}}{f}\right) h_{t j}^{\prime}+G_{1} h_{t j}\right]=0, \\
& a_{i}^{\prime \prime}+\left(\frac{u^{\prime}}{u}+\frac{h^{\prime}}{2 h}\right) a_{i}^{\prime}-\left(\frac{2 q^{2} \phi^{2}}{u}+\frac{k_{z}^{2}}{h u}\right) a_{i}-\frac{A_{z}^{\prime}}{h}\left(h_{i z}^{\prime}-\frac{f^{\prime}}{f} h_{i z}\right) \\
& -\frac{2 i \zeta k_{z}}{\sqrt{h}} \epsilon_{i j}\left(\frac{E_{1}}{u} h_{t j}^{\prime}+H_{1} h_{t j}\right)=0,
\end{aligned}
$$

and constraint equation

$$
h_{i z}^{\prime}-\frac{f^{\prime}}{f} h_{i z}+A_{z}^{\prime} a_{i}-\frac{2 i \zeta k_{z}}{\sqrt{h}} \epsilon_{i j}\left(\frac{u^{\prime}}{u}-\frac{f^{\prime}}{f}\right) A_{z}^{\prime} h_{t j}=0,
$$

where $i, j \in\{x, y\}, \epsilon_{x y}=-\epsilon_{y x}=1$, and the coefficients $C_{1}, D_{1}, E_{1}, F_{1}, G_{1}, H_{1}$ are 


$$
\begin{aligned}
& C_{1}=-\frac{f^{\prime \prime}}{f}-\frac{f^{\prime} h^{\prime}}{2 f h}-\frac{k_{z}^{2}}{h u}, \\
& D_{1}=\frac{u A_{z}^{\prime}}{h}\left(\frac{u^{\prime}}{u}-\frac{f^{\prime}}{f}\right), \\
& E_{1}=-u\left(\frac{u^{\prime}}{u}-\frac{f^{\prime}}{f}\right)\left(\frac{2 h^{\prime}}{h}+\frac{f^{\prime}}{f}\right), \\
& F_{1}=2 u^{\prime \prime \prime}-\frac{2 u f^{\prime \prime \prime}}{f}-\frac{2 u f^{\prime 3}}{f^{3}}+u^{\prime}\left(\frac{2 f^{\prime 2}}{f^{2}}-\frac{4 f^{\prime \prime}}{f}\right)+\frac{4 u f^{\prime} f^{\prime \prime}}{f^{2}}, \\
& G_{1}=\left(\frac{u^{\prime}}{u}-\frac{f^{\prime}}{f}\right)\left(A_{z}^{\prime \prime}-\frac{3 h^{\prime}}{2 h} A_{z}^{\prime}\right), \\
& H_{1}=\left(\frac{u^{\prime}}{u}-\frac{f^{\prime}}{f}\right)\left[\left(\frac{f^{\prime \prime}}{f}-\frac{h^{\prime \prime}}{h}\right)+\frac{3 h^{\prime}}{2 h}\left(\frac{f^{\prime}}{f}+\frac{h^{\prime}}{h}\right)\right] .
\end{aligned}
$$

It is easy to show that (B5) is consistent with (B4) by using the equation of motion for the background fields. Thus, $h_{i z}$ is completely determined by $a_{i}$ and $h_{t j}$.

The above equations can be further simplified as

$$
\begin{array}{r}
h_{t i}^{\prime \prime}+\frac{h^{\prime}}{2 h} h_{t i}^{\prime}+\left(C_{1}-\frac{2 \zeta^{2} k_{z}^{2}}{u} D_{1}^{2}\right) h_{t i}-\frac{2 i \zeta k_{z}}{\sqrt{h}} \epsilon_{i j}\left[E_{1} a_{j}^{\prime}+\left(F_{1}-D_{1} A_{z}^{\prime}\right) a_{j}\right]=0, \\
a_{i}^{\prime \prime}+\left(\frac{u^{\prime}}{u}+\frac{h^{\prime}}{2 h}\right) a_{i}^{\prime}-\left(\frac{2 q^{2} \phi^{2}}{u}+\frac{k_{z}^{2}}{h u}-\frac{A_{z}^{\prime 2}}{h}\right) a_{i}-\frac{2 i \zeta k_{z}}{\sqrt{h}} \epsilon_{i j}\left[\frac{E_{1}}{u} h_{t j}^{\prime}+\left(H_{1}+\frac{D_{1}}{u} A_{z}^{\prime}\right) h_{t j}\right]=0 .
\end{array}
$$

One can solve them order by order,

$$
h_{t i}=h_{t i}^{(0)}+k_{z} h_{t i}^{(1)}+k_{z}^{2} h_{t i}^{(2)}+\ldots, \quad a_{i}=a_{i}^{(0)}+k_{z} a_{i}^{(1)}+k_{z}^{2} a_{i}^{(2)}+\ldots
$$

We consider the sector $h_{t i}^{(0)}=u, a_{i}^{(0)}=0$; thus, $h_{t i}^{(1)}=0$, and the equation for $a_{i}^{(1)}$ is

$$
a_{i}^{(1) \prime \prime}+\left(\frac{u^{\prime}}{u}+\frac{h^{\prime}}{2 h}\right) a_{i}^{(1) \prime}-\left(\frac{2 q^{2} \phi^{2}}{u}-\frac{A_{z}^{\prime 2}}{h}\right) a_{i}^{(1)}-\frac{2 i \zeta}{\sqrt{h}} \epsilon_{i j}\left(\frac{u^{\prime}}{u}-\frac{f^{\prime}}{f}\right) L_{1}=0
$$

with

$$
L_{1}=\left[-\left(\frac{2 h^{\prime}}{h}+\frac{f^{\prime}}{f}\right) h_{t j}^{(0) \prime}+\left(\left(\frac{f^{\prime \prime}}{f}-\frac{h^{\prime \prime}}{h}\right)+\frac{3 h^{\prime}}{2 h}\left(\frac{f^{\prime}}{f}+\frac{h^{\prime}}{h}\right)+\frac{A_{z}^{\prime 2}}{h}\right) h_{t j}^{(0)}\right]
$$

\section{Asymptotic expansion}

Near boundary, the fluctuations can be expanded as

$$
\begin{aligned}
h_{t i} & =h_{t i}^{[0]} r^{2}-h_{t i}^{[0]}\left(\frac{k_{z}^{2}}{4}+\frac{M^{2}}{3}\right)+\frac{\ln r}{r^{2}}\left[\frac{h_{t i}^{[0]}}{144}\left(9 k_{z}^{4}+16 M^{4}+24 M^{4} \lambda+6 M^{2} k_{z}^{2}\right)\right]+\frac{h_{t i}^{[2]}}{4 r^{2}}+\ldots \\
h_{i z} & =h_{i z}^{[0]} r^{2}-h_{i z}^{[0]} \frac{M^{2}}{3}+\frac{\ln r}{r^{2}}\left[\frac{h_{i z}^{[0]}}{18}\left(2 M^{4}+3 M^{4} \lambda\right)+2 a_{i}^{[0]} b M^{2} q^{2}\right]+\frac{h_{i z}^{[2]}}{4 r^{2}}+\ldots \\
a_{i} & =a_{i}^{[0]}-a_{i}^{[0]}\left(M^{2} q^{2}+\frac{k_{z}^{2}}{2}\right) \frac{\ln r}{r^{2}}+\frac{a_{i}^{[2]}}{r^{2}}+\ldots \\
v_{i} & =v_{i}^{[0]}-\frac{k_{z}^{2}}{2} v_{i}^{[0]} \frac{\ln r}{r^{2}}+\frac{v_{i}^{[2]}}{r^{2}}+\ldots
\end{aligned}
$$

where $i \in\{x, y\}$. 


\section{On-shell action}

The renormalized action is given by $S_{\text {ren }}=S+S_{\text {bnd }}$ with

$$
\begin{aligned}
S_{\mathrm{bnd}}= & \frac{1}{\kappa^{2}} \int_{r=r_{\infty}} d^{4} x \sqrt{-\gamma} K-\frac{1}{2 \kappa^{2}} \int_{r=r_{\infty}} d^{4} x \sqrt{-\gamma}\left[6+\frac{1}{2} R+\cdots\right] \\
& +\frac{\log r}{4} \int_{r=r_{\infty}} d^{4} x \sqrt{-\gamma}\left[\mathcal{F}_{\mu \nu} \mathcal{F}^{\mu \nu}+F_{\mu \nu} F^{\mu \nu}+\left|D_{m} \Phi\right|^{2}+\left(\frac{1}{3}+\frac{\lambda}{2}\right)|\Phi|^{4}\right]
\end{aligned}
$$

where $\gamma_{a b}$ is the induced metric on the boundary, and $K$ and $R$ are extrinsic and intrinsic curvatures, respectively. The renormalized on-shell action contains the following terms at quadratic order in fluctuations:

$$
\begin{aligned}
S_{\text {on-shell }} \supset & \int \frac{d k_{z}}{2 \pi} d^{3} x\left[2 a_{x}^{[0]}\left(-k_{z}\right) a_{x}^{[2]}\left(k_{z}\right)+2 a_{y}^{[0]}\left(-k_{z}\right) a_{y}^{[2]}\left(k_{z}\right)-h_{t x}^{[0]}\left(-k_{z}\right) h_{t x}^{[2]}\left(k_{z}\right)-h_{t y}^{[0]}\left(-k_{z}\right) h_{t y}^{[2]}\left(k_{z}\right)\right. \\
& \left.+h_{x z}^{[0]}\left(-k_{z}\right) h_{x z}^{[2]}\left(k_{z}\right)+h_{y z}^{[0]}\left(-k_{z}\right) h_{y z}^{[2]}\left(k_{z}\right)+\mathcal{O}\left(k_{z}^{2}\right)+\text { contact terms }\right],
\end{aligned}
$$

where

$$
\begin{aligned}
\text { contact terms }= & \left(h_{t x}^{[0]}\left(-k_{z}\right) h_{t x}^{[0]}\left(k_{z}\right)+h_{t y}^{[0]}\left(-k_{z}\right) h_{t y}^{[0]}\left(k_{z}\right)\right)\left(4 f_{3}-\frac{7 M^{4}}{36}+2 M O-M_{b}\right) \\
& +\left(h_{x z}^{[0]}\left(-k_{z}\right) h_{x z}^{[0]}\left(k_{z}\right)+h_{y z}^{[0]}\left(-k_{z}\right) h_{y z}^{[0]}\left(k_{z}\right)\right)\left(4 f_{3}-\frac{7 M^{4}}{12}+2 M O-\frac{M_{b}}{3}-\frac{M^{4} \lambda}{2}\right) \\
& +M^{2} q^{2}\left(a_{x}^{[0]}\left(-k_{z}\right) a_{x}^{[0]}\left(k_{z}\right)+a_{y}^{[0]}\left(-k_{z}\right) a_{y}^{[0]}\left(k_{z}\right)\right) \\
& +\left(a_{x}^{[0]}\left(-k_{z}\right) h_{x z}^{[0]}\left(k_{z}\right)+a_{y}^{[0]}\left(-k_{z}\right) h_{y z}^{[0]}\left(k_{z}\right)\right)\left(-b M^{2} q^{2}-2 \xi\right) \\
& -\frac{1}{2} b M^{2} q^{2}\left(h_{x z}^{[0]}\left(-k_{z}\right) a_{x}^{[0]}\left(k_{z}\right)+h_{y z}^{[0]}\left(-k_{z}\right) a_{y}^{[0]}\left(k_{z}\right)\right)+\mathcal{O}\left(k_{z}^{2}\right) .
\end{aligned}
$$

With the sourceless boundary condition for $a_{i}$, i.e., $a_{i}^{[0]}=0$, we normalized the source of $h_{t j}$ to be $h_{t j}^{[0]}=1$, where $i$, $j \in\{x, y\}$ and $i \neq j$; we have

$$
G_{i, t j}^{R}=2 a_{i}^{[2]}
$$

[1] D. E. Kharzeev, The chiral magnetic effect and anomalyinduced transport, Prog. Part. Nucl. Phys. 75, 133 (2014).

[2] K. Landsteiner, Notes on anomaly induced transport, Acta Phys. Pol. B 47, 2617 (2016).

[3] D. E. Kharzeev, J. Liao, S. A. Voloshin, and G. Wang, Chiral magnetic and vortical effects in high-energy nuclear collisions-A status report, Prog. Part. Nucl. Phys. 88, 1 (2016).

[4] M. Kaminski, C. F. Uhlemann, M. Bleicher, and J. SchaffnerBielich, Anomalous hydrodynamics kicks neutron stars, Phys. Lett. B 760, 170 (2016).

[5] E. Shaverin and A. Yarom, An anomalous propulsion mechanism, Nucl. Phys. B928, 268 (2018).

[6] Y.W. Sun and Q. Yang, Negative magnetoresistivity in holography, J. High Energy Phys. 09 (2016) 122.
[7] K. Landsteiner, E. Megias, and F. Pena-Benitez, Gravitational Anomaly and Transport, Phys. Rev. Lett. 107, 021601 (2011).

[8] K. Landsteiner, E. Megias, L. Melgar, and F. Pena-Benitez, Holographic gravitational anomaly and chiral vortical effect, J. High Energy Phys. 09 (2011) 121.

[9] J. Erdmenger, M. Haack, M. Kaminski, and A. Yarom, Fluid dynamics of $R$-charged black holes, J. High Energy Phys. 01 (2009) 055.

[10] N. Banerjee, J. Bhattacharya, S. Bhattacharyya, S. Dutta, R. Loganayagam, and P. Surowka, Hydrodynamics from charged black branes, J. High Energy Phys. 01 (2011) 094.

[11] D. T. Son and P. Surowka, Hydrodynamics with Triangle Anomalies, Phys. Rev. Lett. 103, 191601 (2009). 
[12] Y. Neiman and Y. Oz, Relativistic hydrodynamics with general anomalous charges, J. High Energy Phys. 03 (2011) 023.

[13] S. Golkar and D. T. Son, (Non)-renormalization of the chiral vortical effect coefficient, J. High Energy Phys. 02 (2015) 169.

[14] D. F. Hou, H. Liu, and H. C. Ren, A possible higher order correction to the vortical conductivity in a gauge field plasma, Phys. Rev. D 86, 121703 (2012).

[15] K. Jensen, R. Loganayagam, and A. Yarom, Thermodynamics, gravitational anomalies and cones, J. High Energy Phys. 02 (2013) 088.

[16] G. Basar, D. E. Kharzeev, and I. Zahed, Chiral and Gravitational Anomalies on Fermi Surfaces, Phys. Rev. Lett. 111, 161601 (2013).

[17] K. Jensen, R. Loganayagam, and A. Yarom, Chern-Simons terms from thermal circles and anomalies, J. High Energy Phys. 05 (2014) 110.

[18] M. A. Stephanov and H. U. Yee, No-Drag Frame for Anomalous Chiral Fluid, Phys. Rev. Lett. 116, 122302 (2016).

[19] S. Golkar and S. Sethi, Global anomalies and effective field theory, J. High Energy Phys. 05 (2016) 105.

[20] S. D. Chowdhury and J. R. David, Global gravitational anomalies and transport, J. High Energy Phys. 12 (2016) 116.

[21] P. Glorioso, H. Liu, and S. Rajagopal, Global anomalies, discrete symmetries, and hydrodynamic effective actions, J. High Energy Phys. 01 (2019) 043.

[22] J. Gooth et al., Experimental signatures of the mixed axialgravitational anomaly in the Weyl semimetal NbP, Nature (London) 547, 324 (2017).

[23] J. Gooth, F. Menges, N. Kumar, V. Süß, C. Shekhar, Y. Sun, U. Drechsler, R. Zierold, C. Felser, and B. Gotsmann, Thermal and electrical signatures of a hydrodynamic electron fluid in tungsten diphosphide, Nat. Commun. 9, 4093 (2018).

[24] K. Landsteiner, Y. Liu, and Y.W. Sun, Quantum Phase Transition between a Topological and a Trivial
Semimetal from Holography, Phys. Rev. Lett. 116, 081602 (2016).

[25] K. Landsteiner and Y. Liu, The holographic Weyl semimetal, Phys. Lett. B 753, 453 (2016).

[26] M. Ammon, M. Heinrich, A. Jimenez-Alba, and S. Moeckel, Surface States in Holographic Weyl Semimetals, Phys. Rev. Lett. 118, 201601 (2017).

[27] K. Landsteiner, Y. Liu, and Y. W. Sun, Odd Viscosity in the Quantum Critical Region of a Holographic Weyl Semimetal, Phys. Rev. Lett. 117, 081604 (2016).

[28] G. Grignani, A. Marini, F. Pena-Benitez, and S. Speziali, AC conductivity for a holographic Weyl semimetal, J. High Energy Phys. 03 (2017) 125.

[29] C. Copetti, J. Fernandez-Pendas, and K. Landsteiner, Axial Hall effect and universality of holographic Weyl semimetals, J. High Energy Phys. 02 (2017) 138.

[30] M. Ammon, M. Baggioli, A. Jimenez-Alba, and S. Moeckel, A smeared quantum phase transition in disordered holography, J. High Energy Phys. 04 (2018) 068.

[31] M. Baggioli, B. Padhi, P. W. Phillips, and C. Setty, Conjecture on the butterfly velocity across a quantum phase transition, J. High Energy Phys. 07 (2018) 049.

[32] Y. Liu and Y. W. Sun, Topological invariants for holographic semimetals, J. High Energy Phys. 10 (2018) 189.

[33] Y. Liu and J. Zhao, Weyl semimetal/insulator transition from holography, J. High Energy Phys. 12 (2018) 124.

[34] K. Landsteiner, Y. Liu, and Y. W. Sun, Holographic topological semimetals, arXiv:1911.07978.

[35] S. Lin and L. Yang, Mass correction to chiral vortical effect and chiral separation effect, Phys. Rev. D 98, 114022 (2018).

[36] A. Flachi and K. Fukushima, Chiral vortical effect with finite rotation, temperature, and curvature, Phys. Rev. D 98, 096011 (2018).

[37] C. Copetti and K. Landsteiner, Anomalous Hall viscosity at the Weyl semimetal/insulator transition, Phys. Rev. B 99, 195146 (2019). 